\title{
INCLUSION OF MECHANICAL DAMPERS IN THE MULTIMODAL FLUTTER ANALYSIS OF SLENDER STRUCTURES
}

\author{
JUAN QUINTELA, JOSÉ A. JURADO, CARLOS RAPELA, \\ ANTONIO J. ÁLVAREZ \& SANTIAGO HERNÁNDEZ \\ School of Civil Engineering, Universidade da Coruña, Spain
}

\begin{abstract}
Sometimes slender structures are reinforced with mechanical dampers to reduce the vibrations caused by aeroelastic phenomena like flutter. However the formulation of flutter analysis only considers the classical damping ratio to take into account the structural damping. This paper explains the procedure used for adding mechanical dampers with a known constant to the analysis software FLAS. This code was developed at Universidade da Coruña to calculate the critical wind speed for flutter instability. An example of a solar tracker with two rows of flat panels is shown. In this slender structure two mechanical dampers are used to reduce the vibrations caused by the wind in structure interaction. The solar tracker has been studied for five different positions of the angle of attack. Results of flutter speed for several values of the dampers constant and global structural damping ratio are presented.

Keywords: multimodal flutter analysis, mechanical dampers, solar tracker.
\end{abstract}

\section{INTRODUCTION}

Multimodal analysis software FLAS was developed in 2001 in the Universidade da Coruña in order to evaluate the critical wind speed of long span bridges. This program was used in different researches for the optimization of long span bridges [1], [2] and also for reliability based design optimization (RBDO) [3]. In these works only the structural damping was considered during the analysis, introduced as a constant value for each natural vibration mode. This paper includes a modification of the software to model mechanical dampers which add a local effect to the global damping of the structure.

The problem consists in solving the dynamic equilibrium equation considering the selfexcited forces caused by fluid-structure interactions.

\section{MULTIMODAL FLUTTER ANALYSIS WITH DAMPERS}

The dynamic equilibrium equation considering self-excited forces can be written as

$$
\mathbf{M u ̈}+\mathbf{C u}+\mathbf{K u}=\mathbf{f}_{a},
$$

where $\mathbf{M}, \mathbf{C}$ and $\mathbf{K}$ are the mass damping and stiffness matrix respectively and $\mathbf{u}, \dot{\mathbf{u}}$ and $\ddot{\mathbf{u}}$ are the displacement, velocity and acceleration vectors of the structure. The aeroelastic forces are a linear combination of the displacements and velocities and can be expressed in terms of the stiffness and damping matrix where the load vector acts on each element $i$ as

$$
\mathbf{f}_{a, i}=\mathbf{K}_{a, i} \mathbf{u}_{i}+\mathbf{C}_{a, i} \dot{\mathbf{u}}_{i},
$$

where the subscript $a$ refers to the aeroelastic origin of the terms. Loads are distributed along the bar element so each node carries out half of the load. Therefore eqn (2) can be written in matrix form for either end $i=1,2$ as 


$$
\begin{gathered}
\left(\begin{array}{c}
F_{x} \\
F_{y} \\
F_{z} \\
M_{x} \\
M_{y} \\
M_{z}
\end{array}\right)=\frac{l_{e}}{2}\left(\begin{array}{c}
0 \\
p_{y} \\
p_{z} \\
m_{x} \\
0 \\
0
\end{array}\right)=\frac{l_{e}}{2}\left(\begin{array}{cccccc}
0 & 0 & 0 & 0 & 0 & 0 \\
0 & p K^{2} P_{4}^{*} & p K^{2} P_{6}^{*} & -p B K^{2} P_{3}^{*} & 0 & 0 \\
0 & -p K^{2} H_{6}^{*} & p K^{2} H_{4}^{*} & p B K^{2} H_{3}^{*} & 0 & 0 \\
0 & -p B K^{2} A_{6}^{*} & p B K^{2} A_{4}^{*} & p B^{2} K^{2} A_{3}^{*} & 0 & 0 \\
0 & 0 & 0 & 0 & 0 & 0 \\
0 & 0 & 0 & 0 & 0 & 0
\end{array}\right)\left(\begin{array}{c}
u_{i} \\
v_{i} \\
w_{i} \\
\phi_{x i} \\
\phi_{y i} \\
\phi_{z i}
\end{array}\right)+ \\
\frac{l_{e}}{2}\left(\begin{array}{cccccc}
0 & 0 & 0 & 0 & 0 & 0 \\
0 & p B K P_{1}^{*} / U & -p B K P_{5}^{*} / U & -p B^{2} K P_{2}^{*} / U & 0 & 0 \\
0 & -p B K H_{5}^{*} / U & p B K H_{1}^{*} / U & p B^{2} K H_{2}^{*} / U & 0 & 0 \\
0 & -p B^{2} K A_{5}^{*} / U & p B^{2} K A_{1}^{*} / U & p B^{3} K A_{2}^{*} / U & 0 & 0 \\
0 & 0 & 0 & 0 & 0 & 0 \\
0 & 0 & 0 & 0 & 0 & 0
\end{array}\right)\left(\begin{array}{c}
\dot{u}_{i} \\
\dot{v}_{i} \\
\dot{w}_{i} \\
\dot{\phi}_{x i} \\
\dot{\phi}_{y i} \\
\dot{\phi}_{z i}
\end{array}\right)
\end{gathered}
$$

where $B$ is the width, $\rho$ is the air density, $\mathrm{U}$ is the mean wind velocity, $\mathrm{K}=\mathrm{B} \omega / \mathrm{U}$ the reduced frequency with $\omega$ the circular frequency and $H_{\mathrm{i}}{ }^{*}, P_{\mathrm{i}}{ }^{*}, A_{\mathrm{i}}{ }^{*}(i=1-6)$ are the frequency dependant flutter derivatives.

It is possible to define a load vector acting on each element of the deck discretization (Fig. 1). The forces acting on the element nodes (Fig. 2) are related to its movements and speed and each node carries half the loads distributed along the element.

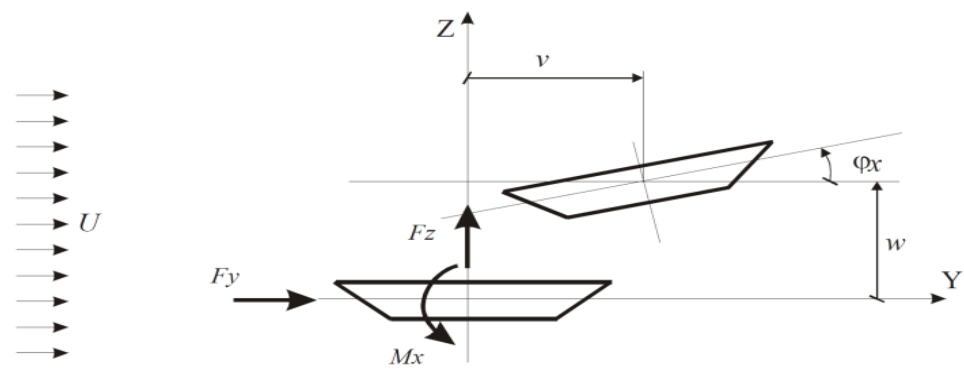

Figure 1: Aeroelastic forces and sign criteria.

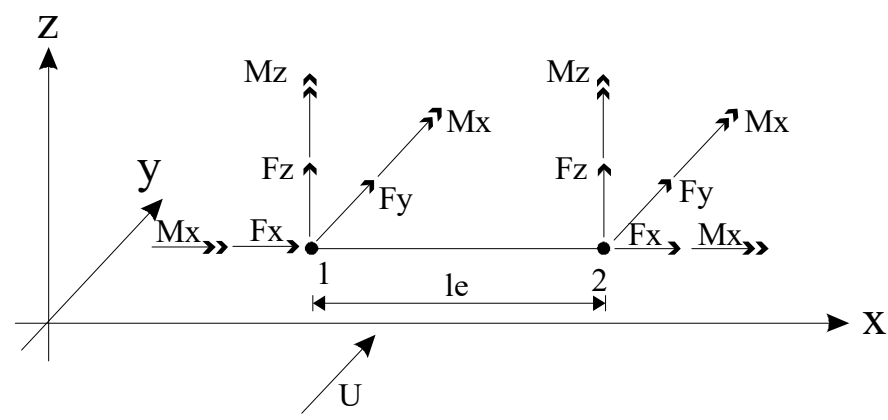

Figure 2: Aeroelastic forces acting on bar elements along the deck. 
Assembling all the bar elements that constitute the deck with all the vectors $\mathbf{f}_{a e}$ and all matrices $\mathbf{K}_{a e}$ and $\mathbf{C}_{a e}$ a global vector of aeroelastic forces $\mathbf{f}_{a}$ and the aeroelastic global matrices $\mathbf{K}_{a}$ and $\mathbf{C}_{a}$ are obtained. The following relationship is achieved for the global vector of aeroelastic forces

$$
\mathbf{f}_{a}=\mathbf{K}_{a} \mathbf{u}+\mathbf{C}_{a} \dot{\mathbf{u}} .
$$

In order to include the effect of mechanical dampers a new mechanical damping matrix $\mathbf{C}_{m}$ is defined

$$
\mathbf{f}_{m, i}=\left(\begin{array}{l}
F_{x} \\
F_{y} \\
F_{z} \\
M_{x} \\
M_{y} \\
M_{z}
\end{array}\right)_{i}=\left(\begin{array}{cccccc}
c_{u} & 0 & 0 & 0 & 0 & 0 \\
0 & c_{v} & 0 & 0 & 0 & 0 \\
0 & 0 & c_{w} & 0 & 0 & 0 \\
0 & 0 & 0 & c_{\varphi x} & 0 & 0 \\
0 & 0 & 0 & 0 & c_{\varphi y} & 0 \\
0 & 0 & 0 & 0 & 0 & c_{\varphi z}
\end{array}\right)\left(\begin{array}{l}
\dot{u} \\
\dot{v} \\
\dot{w} \\
\dot{\varphi}_{x} \\
\dot{\varphi}_{y} \\
\dot{\varphi}_{z}
\end{array}\right)=\mathbf{C}_{m} \dot{\mathbf{u}}_{i}
$$

where $c_{\varphi}$ is the damper constant which connects the moment and the angular velocity. The dampers of the solar tracker apply a moment which is dependent on the distance to the axis of the solar tracker as shown in Fig. 3.

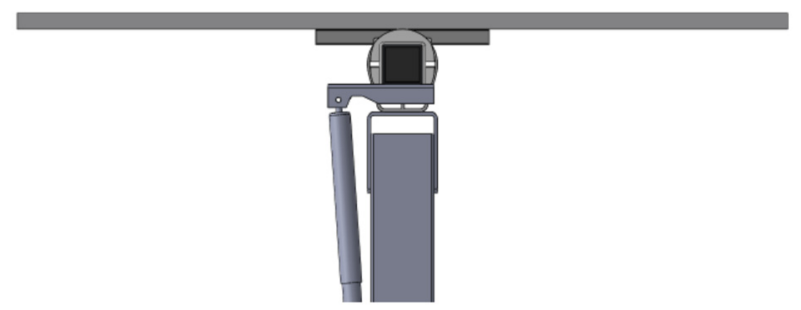

Figure 3: Scheme of the damper.

Knowing the damping constant and the distance $a$ of the damper to the axis of the solar tracker the value of $c_{\varphi}$ can be obtained as

$$
M_{x}=F a=c_{\varphi} \dot{\varphi}_{x}=c_{\varphi} \frac{\dot{w}}{a} \Rightarrow F=\frac{c_{\varphi}}{a^{2}} \dot{w}=c_{w} \dot{w} \Rightarrow c_{\varphi}=c_{w} a^{2} .
$$

Introducing eqn (4) in eqn (1) and considering the new matrix $\mathbf{C}_{m}$ and arranging terms of aeroelastic forces to the left side of the equality the system of equations governing the dynamic behaviour while withstanding forces of aeroelastic origin is obtained

$$
\mathbf{M} \ddot{\mathbf{u}}+\left(\mathbf{C}+\mathbf{C}_{m}-\mathbf{C}_{a}\right) \dot{\mathbf{u}}+\left(\mathbf{K}-\mathbf{K}_{a}\right) \mathbf{u}=\mathbf{0} .
$$

Matrix $\mathbf{C}$ contains the classical structural damping ratio, matrix $\mathbf{C}_{m}$ links the forces that the mechanical dampers apply with the velocities of the nodes where the forces act, and matrix $\mathbf{C}_{a}$ represents the aeroelastic damping. Expressing displacements as a linear combination of $m$ vibration modes grouped in the modal matrix $\boldsymbol{\Phi}=\mathbf{u q}$, where $\mathbf{q}$ is the participation vector the follow relationship is obtained

$$
\mathbf{I} \ddot{\mathbf{q}}+\mathbf{C}_{R} \dot{\mathbf{q}}+\mathbf{K}_{R} \mathbf{q}=\mathbf{0} .
$$


Assuming a decreasing oscillatory solution for the vector $\mathbf{q}=\mathbf{w e}^{\mu t}$ where $\mathbf{w}$ and $\mu$ are complex values and using the equality $-\mu \mathbf{I} \mathbf{w}+\mu \mathbf{I} \mathbf{w}=\mathbf{0}$, eqn (8) can be written as an eigenproblem

$$
(\boldsymbol{A}-\mu \boldsymbol{I}) \boldsymbol{w}_{\mu} e^{\mu t}=\mathbf{0} \text { where } \boldsymbol{w}_{\mu}=\left(\begin{array}{c}
\boldsymbol{\mu} \boldsymbol{w} \\
\boldsymbol{w}
\end{array}\right) \text { and } \mathbf{A}=\left(\begin{array}{cc}
-\boldsymbol{C}_{R} & -\boldsymbol{K}_{R} \\
\boldsymbol{I} & \mathbf{0}
\end{array}\right) .
$$

The solution to the above eigen-problem is $\mu_{i}=\alpha_{i}+\mathrm{i} \beta_{i},(i=1, \ldots 2 m)$ where $\alpha$ is related to structural damping and $\beta$ is damping frequency [4]. Matrix $\mathbf{A}$ depends on flutter derivatives, which are functions of the reduced frequency. However, this frequency remains unknown until eigenvalue problems are solved so an iterative procedure must be employed. The critical condition for the flutter phenomenon corresponds with the lowest wind speed. Thus, a complex eigenvalue is obtained whose real part is null; in other words, it is at the transition between positive damping (decreasing oscillations) and a negative one (oscillations of growing amplitude).

\section{APPLICATION TO A SOLAR TRACKER}

A model of a solar tracker with flat panels situated on a girder supported by piers and moved by a motor in the middle is analysed to study the flutter phenomena. Two configurations of the solar tracker with different dimensions have been considered in the analysis.

The structural models shown in Fig. 4 have been defined in SAP2000 using bar elements for the structural parts and shell elements for the photovoltaic panels. Each model have 2,846 nodes, 3,325 bar elements and 360 shell elements and two lines of panels with connecting rod mechanism have been modelled. The solar trackers have mechanical dampers in the two second outermost piers that only act in the torsional degree of freedom. So in this case $c_{\varphi \mathrm{x}}$ is the only defined damping constant in matrix $\mathbf{C}_{\mathrm{m}}$.

Different angles of attack of the solar tracker have been studied, hence one model have been defined for each case. Fig. 5 shows the models used for $\pm 10^{\circ}, \pm 20^{\circ}, \pm 35^{\circ}$ and $\pm 60^{\circ}$.
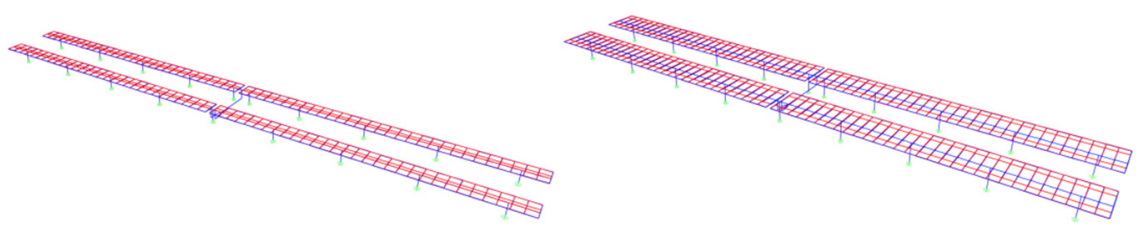

Figure 4: Structural models of the solar trackers for configuration 1 (left) and configuration 2 (right).
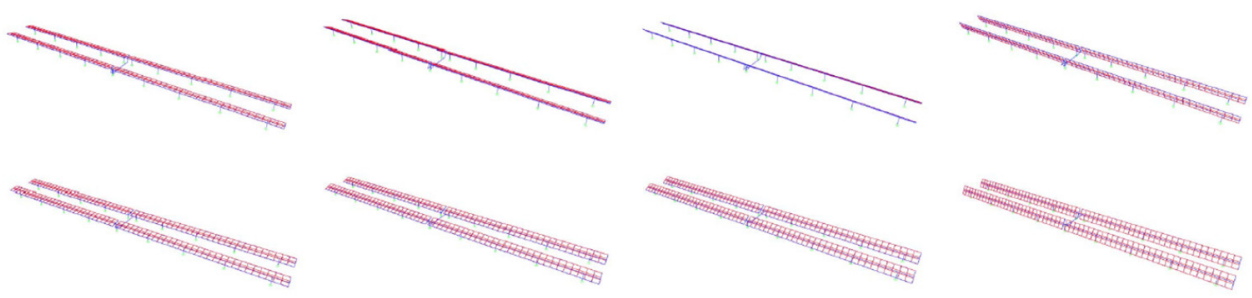

Figure 5: Structural models negative angles of attack $-10^{\circ},-20^{\circ},-35^{\circ},-60^{\circ}$ (top) and for positive angles of attack $+10^{\circ},+20^{\circ},+35^{\circ},+60^{\circ}$ (bottom). 
A modal analysis has been carried out in order to obtain the natural modes and frequencies for each model. Figs 6 and 7 show the first 30 modes obtained in the analysis for both configurations. For each mode the left half of the graph represents the normalized displacement of the windward line of the solar tracker while the right half represents the normalized displacement of the leeward line.

The first four modes are torsional for both configurations and for all the angles of attack, corresponding to the first symmetrical and non-symmetrical modes for each line. All modal shapes are similar for different angles of attack, although more torsional modes for the first modal frequencies tend to appear when increasing the angle of the solar tracker.
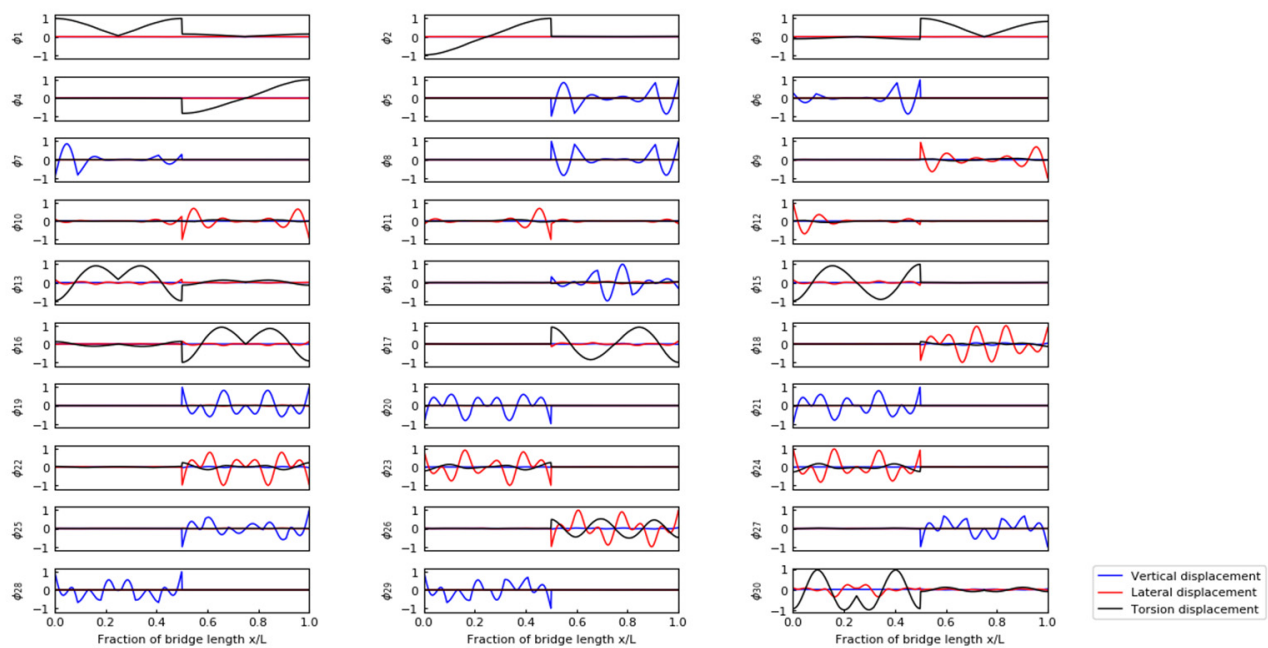

Figure 6: Modal shapes normalized in displacement for $0^{\circ}$ for configuration 1.
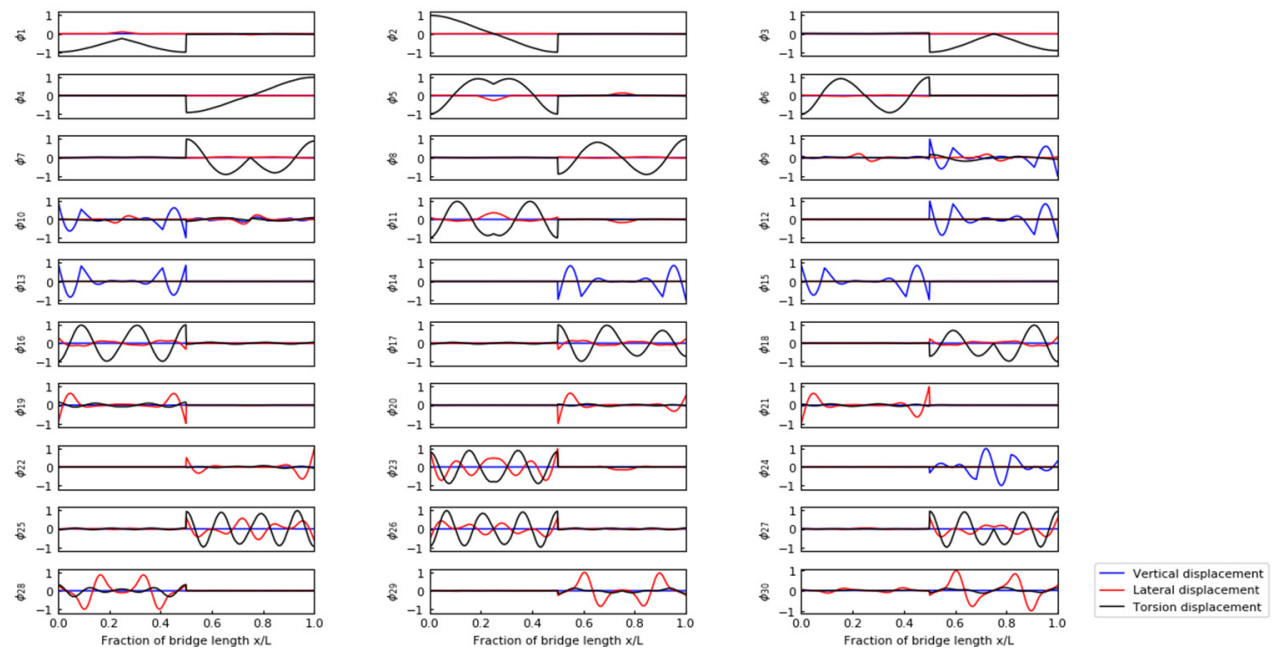

Figure 7: Modal shapes normalized in displacement for $0^{\circ}$ for configuration 2. 
Tables 1 and 2 show the first 10 natural frequencies normalized to the first frequency for both configurations and each angle of attack. Natural frequencies are very similar for the different angles of attack. The first frequencies of the configuration 2 are approximately half of the configuration 1 , which is expected given that the solar tracker of the configuration 2 has double the width of configuration 1 .

Table 1: First natural frequencies normalized for configuration 1.

\begin{tabular}{|c|c|c|c|c|c|c|c|c|c|}
\hline Frequency & $0^{\circ}$ & $10^{\circ}$ & $-10^{\circ}$ & $20^{\circ}$ & $-20^{\circ}$ & $35^{\circ}$ & $-35^{\circ}$ & $60^{\circ}$ & $-60^{\circ}$ \\
\hline$\omega_{1}$ & 1.000 & 1.000 & 1.000 & 1.000 & 1.000 & 1.000 & 1.000 & 1.000 & 1.000 \\
\hline$\omega_{2}$ & 1.038 & 1.032 & 1.033 & 1.033 & 1.034 & 1.035 & 1.038 & 1.048 & 1.060 \\
\hline$\omega_{3}$ & 1.040 & 1.035 & 1.035 & 1.034 & 1.035 & 1.036 & 1.038 & 1.048 & 1.060 \\
\hline$\omega_{4}$ & 1.040 & 1.036 & 1.036 & 1.035 & 1.035 & 1.036 & 1.039 & 1.048 & 1.061 \\
\hline$\omega_{5}$ & 2.036 & 2.029 & 2.029 & 2.026 & 2.027 & 2.029 & 2.034 & 2.054 & 2.077 \\
\hline$\omega_{6}$ & 2.040 & 2.031 & 2.031 & 2.028 & 2.030 & 2.031 & 2.036 & 2.056 & 2.078 \\
\hline$\omega_{7}$ & 2.040 & 2.069 & 2.070 & 2.145 & 2.148 & 2.236 & 2.239 & 2.243 & 2.264 \\
\hline$\omega_{8}$ & 2.041 & 2.072 & 2.073 & 2.148 & 2.151 & 2.239 & 2.243 & 2.246 & 2.266 \\
\hline$\omega_{9}$ & 2.535 & 2.525 & 2.525 & 2.523 & 2.525 & 2.531 & 2.536 & 2.564 & 2.590 \\
\hline$\omega_{10}$ & 2.545 & 2.534 & 2.533 & 2.530 & 2.531 & 2.536 & 2.543 & 2.573 & 2.600 \\
\hline
\end{tabular}

Table 2: First natural frequencies normalized for configuration 2.

\begin{tabular}{|c|c|c|c|c|c|c|c|c|c|}
\hline Frequency & $0^{\circ}$ & $10^{\circ}$ & $-10^{\circ}$ & $20^{\circ}$ & $-20^{\circ}$ & $35^{\circ}$ & $-35^{\circ}$ & $60^{\circ}$ & $-60^{\circ}$ \\
\hline$\omega_{1}$ & 1.000 & 1.000 & 1.000 & 1.000 & 1.000 & 1.000 & 1.000 & 1.000 & 1.000 \\
\hline$\omega_{2}$ & 1.202 & 1.161 & 1.161 & 1.166 & 1.175 & 1.185 & 1.203 & 1.264 & 1.345 \\
\hline$\omega_{3}$ & 1.202 & 1.161 & 1.161 & 1.166 & 1.175 & 1.185 & 1.203 & 1.264 & 1.345 \\
\hline$\omega_{4}$ & 1.203 & 1.161 & 1.161 & 1.166 & 1.175 & 1.186 & 1.205 & 1.264 & 1.345 \\
\hline$\omega_{5}$ & 2.814 & 2.798 & 2.798 & 2.801 & 2.807 & 2.812 & 2.822 & 2.881 & 2.961 \\
\hline$\omega_{6}$ & 3.360 & 3.247 & 3.247 & 3.262 & 3.287 & 3.315 & 3.367 & 3.538 & 3.764 \\
\hline$\omega_{7}$ & 3.360 & 3.247 & 3.247 & 3.262 & 3.287 & 3.315 & 3.367 & 3.538 & 3.764 \\
\hline$\omega_{8}$ & 3.362 & 3.248 & 3.248 & 3.263 & 3.289 & 3.316 & 3.369 & 3.538 & 3.764 \\
\hline$\omega_{9}$ & 3.665 & 3.537 & 3.537 & 3.552 & 3.581 & 3.606 & 3.665 & 3.846 & 4.094 \\
\hline$\omega_{10}$ & 3.805 & 3.673 & 3.673 & 3.687 & 3.719 & 3.745 & 3.806 & 3.997 & 4.252 \\
\hline
\end{tabular}

\section{FLUTTER DERIVATIVES}

Flutter derivatives have been obtained experimentally by aeroelastic tests in wind tunnel [5]. Two sectional models have been built for each configuration. Each sectional model represents a line of panels. Wind tunnel tests were carried out with an increment of $0.33 \mathrm{~m} / \mathrm{s}$ in wind velocity for each angle of attack. Two set ups were considered for obtaining the flutter derivatives. Firstly, one line of panels is supported by springs and tested in order to acquire the flutter derivatives for the windward position (single) as shown in Fig. 8. Secondly, two sectional models are situated in the test chamber; the windward model is fixed without movements, while the leeward model is supported by springs and tested to obtain the flutter derivatives for the leeward position (wake). Fig. 9 shows this last case. 

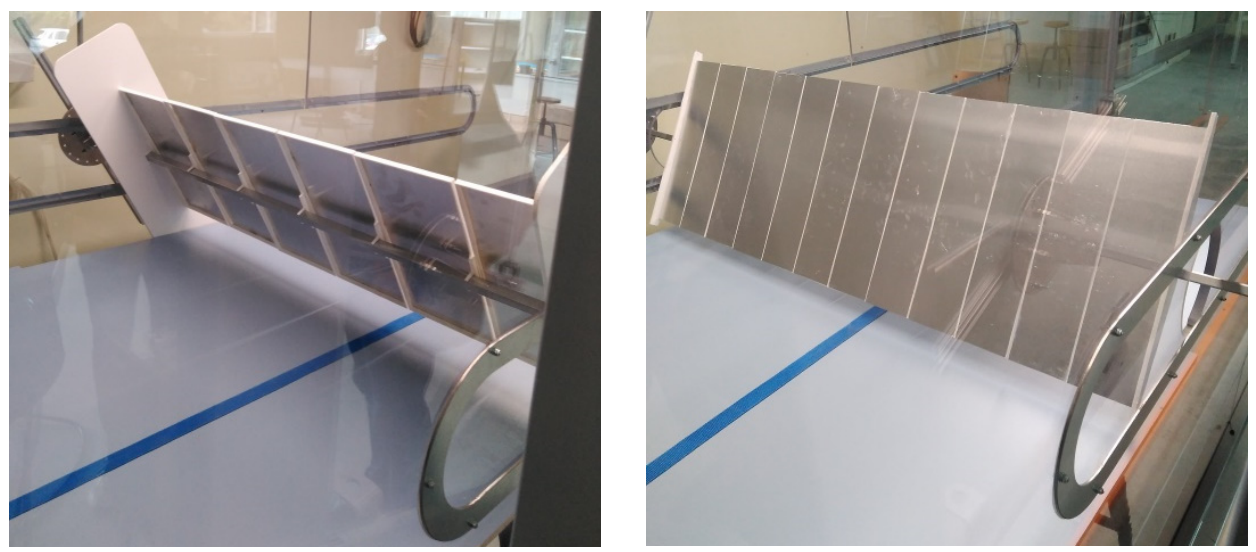

Figure 8: Single set up of the solar tracker for configuration 1 (left) and configuration 2 (right).
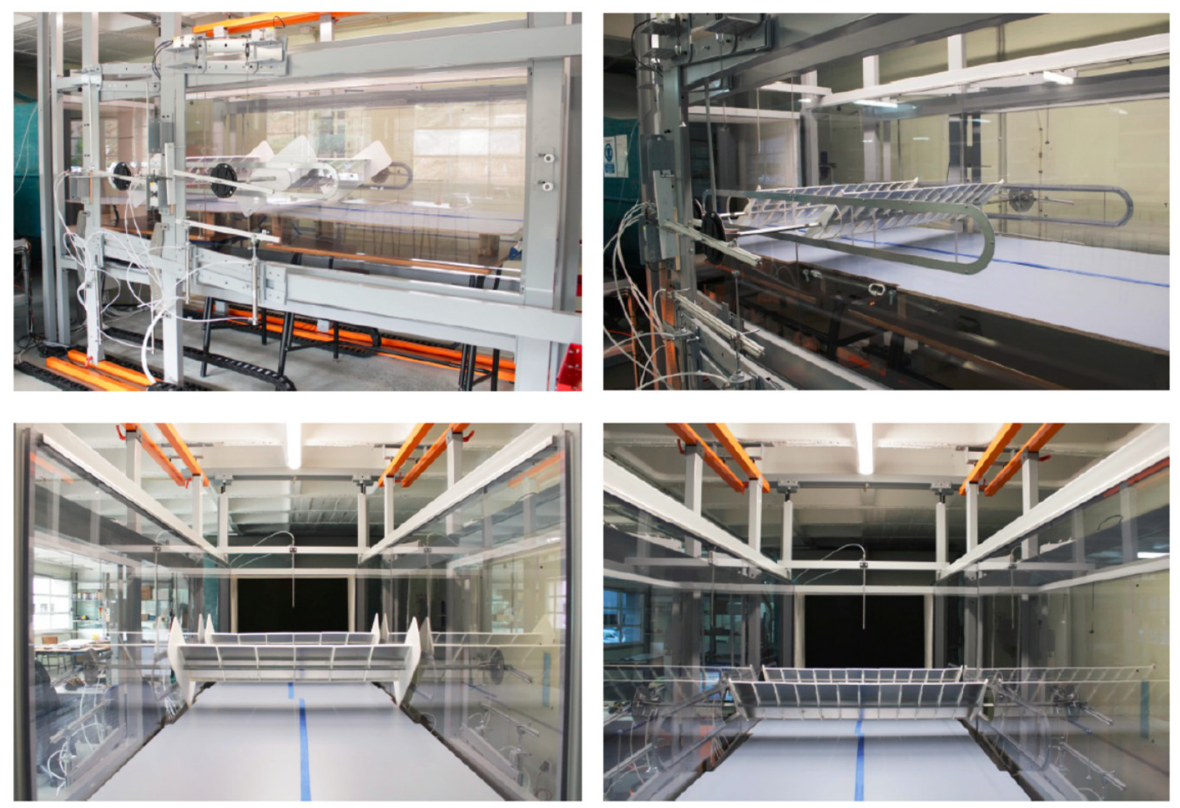

Figure 9: Wake set up of the solar tracker for configuration 1 (left) and configuration 2 (right).

The set up for the wind tunnel tests were in free vibration. This method for obtaining the flutter derivatives consist in supporting the model with springs, allowing the movement in three degrees of freedom, vertical $w$, lateral $v$, and rotation $\varphi_{x}$. Increasing the wind velocity and considering the stiffness of the springs a wide range of reduced frequencies can be cover. The dynamic equation of the sectional model in the wind tunnel is

$$
\mathbf{M u}+\mathbf{C} \dot{\mathbf{u}}+\mathbf{K u}=\mathbf{f}_{a}=\mathbf{C}_{a} \dot{\mathbf{u}}+\mathbf{K}_{a} \mathbf{u},
$$


where $\mathrm{M}, \mathrm{C}$ and $\mathrm{K}$ are respectively the mass, dampening and stiffness matrixes which are dependent on the mass $m$, the inertia of the model $I$ and the spring constants. $\mathrm{f}_{\mathrm{a}}$ is the vector of aeroelastic forces and can be written as a function of the aeroelastic matrixes. Eqn (9) can be written as

$$
\ddot{\mathbf{u}}+\mathbf{C}_{m} \dot{\mathbf{u}}+\mathbf{K}_{m} \mathbf{u}=\mathbf{0},
$$

where $\mathbf{C}_{m}=\mathbf{M}^{-\mathbf{1}}\left(\mathbf{C}-\mathbf{C}_{a}\right)$ and $\mathbf{K}_{m}=\mathbf{M}^{-\mathbf{1}}\left(\mathbf{K}-\mathbf{K}_{a}\right)$. In order to obtain the flutter derivatives the terms of these matrixes have to be calculated. This is achieved by a numerical method of parameter identification which determines the frequency and dampening of the free vibration response. In this case a modified Ibrahim time domain method was used [6], [7]. With the aforementioned parameters is possible to construct the terms of the matrixes. Denominating $K_{i j}^{\text {eff }}$ and $C_{i j}$ eff the stiffness and dampening terms with wind and $K_{i j}{ }^{\text {eech }}$ and $C_{i j}{ }^{\text {mech }}$ the stiffness and dampening terms without wind, the flutter derivatives can be obtained with the following expressions

$$
\begin{array}{ll}
P_{1}^{*}(K)=-\frac{2 m}{\rho B^{2} \omega_{1}}\left(\overline{\mathrm{C}}_{11}^{e f}-\overline{\mathrm{C}}_{11}^{m e c h}\right) & P_{2}^{*}(K)=-\frac{2 m}{\rho B^{3} \omega_{3}}\left(\overline{\mathrm{C}}_{13}^{e f}-\overline{\mathrm{C}}_{13}^{m e c h}\right), \\
P_{3}^{*}(K)=+\frac{2 m}{\rho B^{3} \omega_{3}^{2}}\left(\bar{K}_{13}^{e f}-\overline{\mathrm{K}}_{13}^{m e c h}\right) & P_{4}^{*}(K)=-\frac{2 m}{\rho B^{2} \omega_{1}^{2}}\left(\overline{\mathrm{K}}_{11}^{e f}-\overline{\mathrm{K}}_{11}^{m e c h}\right), \\
P_{5}^{*}(K)=+\frac{2 m}{\rho B^{2} \omega_{2}}\left(\overline{\mathrm{C}}_{12}^{e f}-\overline{\mathrm{C}}_{12}^{m e c h}\right) & P_{6}^{*}(K)=+\frac{2 m}{\rho B^{2} \omega_{2}^{2}}\left(\overline{\mathrm{K}}_{12}^{e f}-\overline{\mathrm{K}}_{12}^{m e c h}\right), \\
H_{1}^{*}(K)=-\frac{2 m}{\rho B^{2} \omega_{2}}\left(\overline{\mathrm{C}}_{22}^{e f}-\overline{\mathrm{C}}_{22}^{m e c h}\right) & H_{2}^{*}(K)=-\frac{2 m}{\rho B^{3} \omega_{3}}\left(\overline{\mathrm{C}}_{23}^{e f}-\overline{\mathrm{C}}_{23}^{m e c h}\right), \\
H_{3}^{*}(K)=-\frac{2 m}{\rho B^{3} \omega_{3}^{2}}\left(\bar{K}_{23}^{e f}-\overline{\mathrm{K}}_{23}^{m e c h}\right) & H_{4}^{*}(K)=-\frac{2 m}{\rho B^{2} \omega_{2}^{2}}\left(\overline{\mathrm{K}}_{22}^{e f}-\overline{\mathrm{K}}_{22}^{m e c h}\right), \\
H_{5}^{*}(K)=+\frac{2 m}{\rho B^{2} \omega_{1}}\left(\overline{\mathrm{C}}_{21}^{e f}-\overline{\mathrm{C}}_{21}^{m e c h}\right) & H_{6}^{*}(K)=+\frac{2 m}{\rho B^{2} \omega_{1}^{2}}\left(\overline{\mathrm{K}}_{21}^{e f}-\overline{\mathrm{K}}_{21}^{m e c h}\right), \\
A_{1}^{*}(K)=-\frac{2 I}{\rho B^{3} \omega_{2}}\left(\overline{\mathrm{C}}_{32}^{e f}-\overline{\mathrm{C}}_{32}^{m e c h}\right) & A_{2}^{*}(K)=-\frac{2 I}{\rho B^{4} \omega_{3}}\left(\overline{\mathrm{C}}_{33}^{e f}-\overline{\mathrm{C}}_{33}^{m e c h}\right), \\
A_{3}^{*}(K)=-\frac{2 I}{\rho B^{4} \omega_{3}^{2}}\left(\bar{K}_{33}^{e f}-\overline{\mathrm{K}}_{33}^{m e c h}\right) & A_{4}^{*}(K)=-\frac{2 I}{\rho B^{3} \omega_{2}^{2}}\left(\overline{\mathrm{K}}_{32}^{e f}-\overline{\mathrm{K}}_{32}^{m e c h}\right), \\
A_{5}^{*}(K)=+\frac{2 I}{\rho B^{3} \omega_{1}}\left(\overline{\mathrm{C}}_{31}^{e f}-\overline{\mathrm{C}}_{31}^{m e c h}\right) & A_{6}^{*}(K)=+\frac{2 I}{\rho B^{3} \omega_{1}^{2}}\left(\overline{\mathrm{K}}_{31}^{e f}-\overline{\mathrm{K}}_{31}^{m e c h}\right) .
\end{array}
$$

The small increment of wind velocity used in the wind tunnel tests means the flutter derivatives obtained have high precision with a great number of data points. A post process of the values obtained in wind tunnel has been carried out to introduce the values of the flutter derivatives in code FLAS, reducing the number of points used to define the tendency of the curve as shown in Figs 10 and 11. In Figs 12 and 13 a comparison between the values of the single flutter derivatives and the wake flutter derivatives used in code FLAS is shown for two of the cases analyzed. 

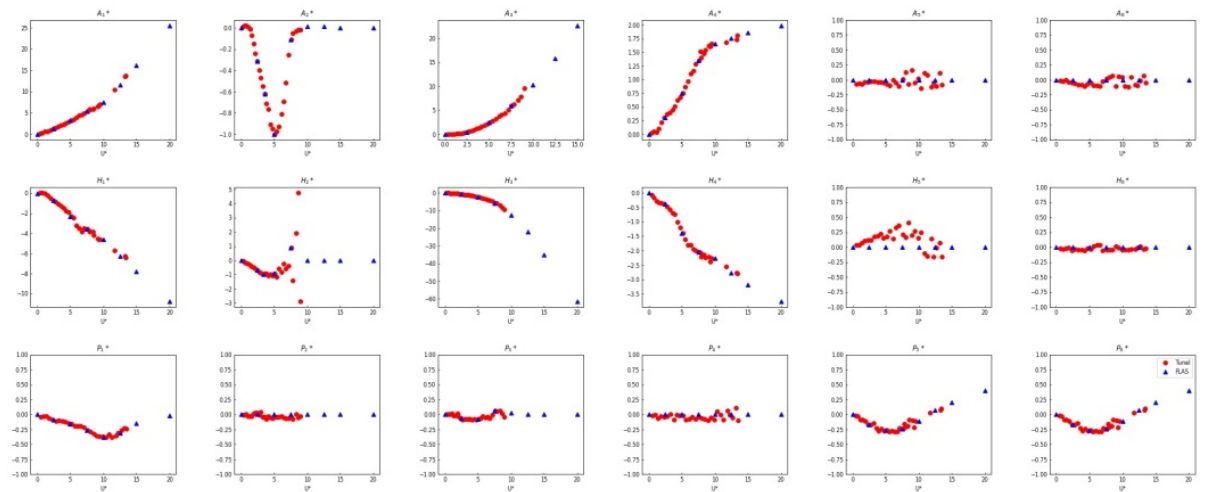

Figure 10: Experimental values (red) and FLAS values (blue) of the single flutter derivatives for the configuration 1 and $0^{\circ}$ angle of attack.
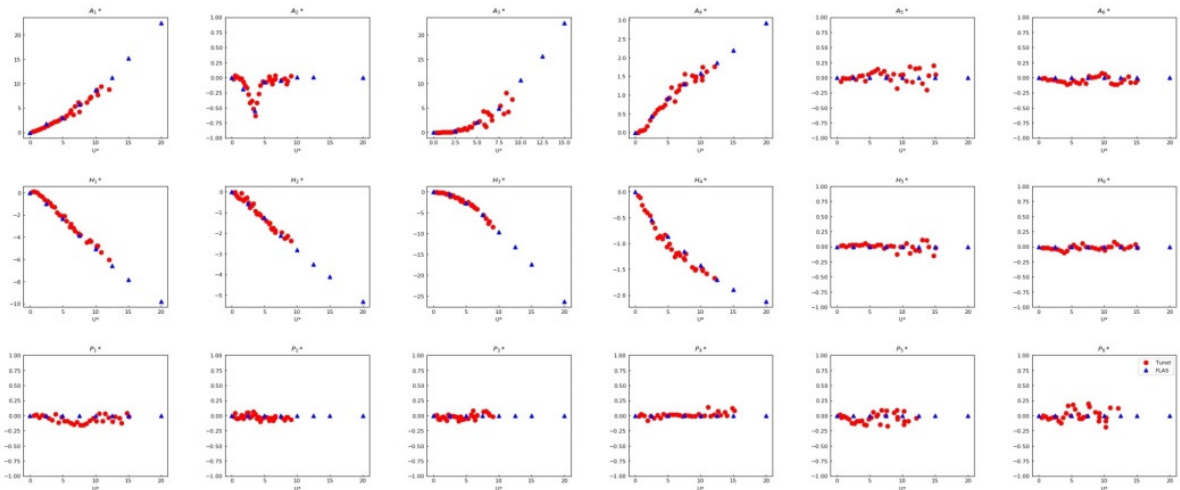

Figure 11: Experimental values (red) and FLAS values (blue) of the wake flutter derivatives for the configuration 1 and $0^{\circ}$ angle of attack.
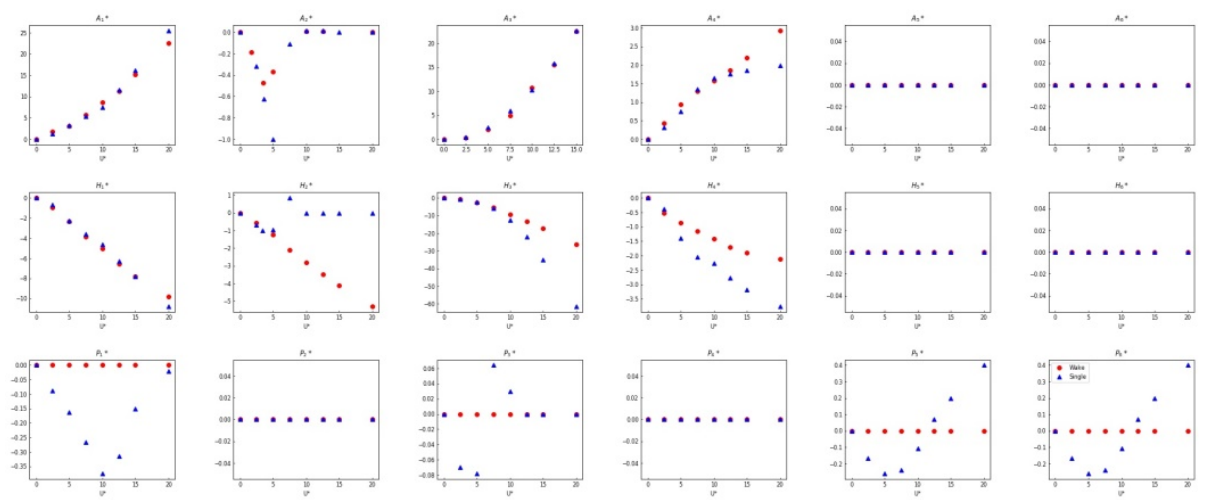

Figure 12: FLAS values for the wake flutter derivatives (red) and FLAS values for the single flutter derivatives (blue) for the configuration 2 and $0^{\circ}$ angle of attack. 


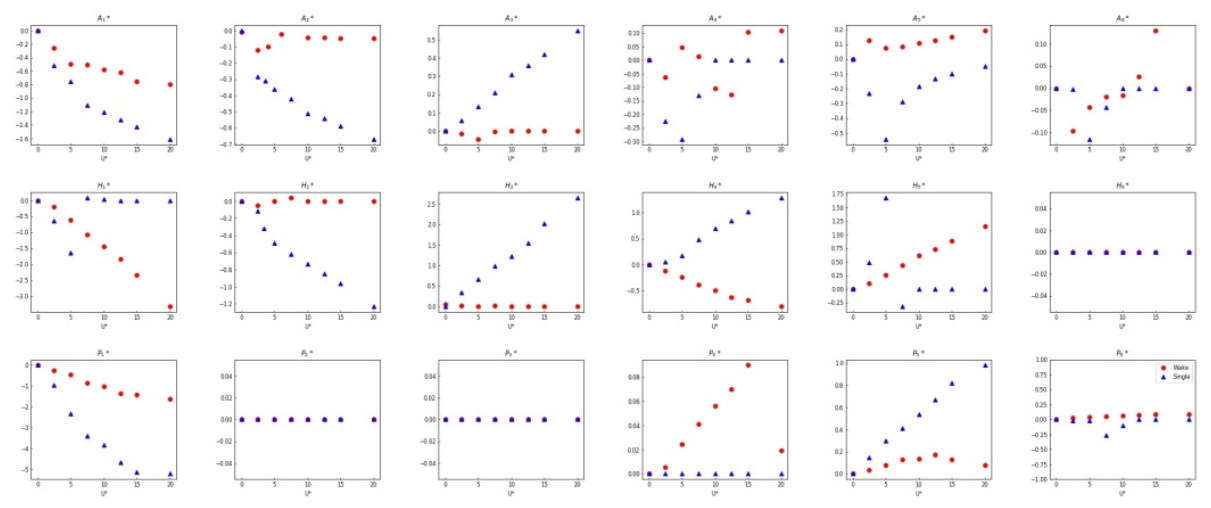

Figure 13: FLAS values for the wake flutter derivatives (red) and FLAS values for the single flutter derivatives (blue) for the configuration 2 and $60^{\circ}$ angle of attack.

\section{RESULTS OF FLUTTER SPEED AND CONCLUSIONS}

The critical flutter speed was calculated using the matrix formulation explained in point 2 . Several analyses were executed with different combinations of natural modes. The analyses were performed with a dampening of $2 \%$ for the lateral modes $\left(\xi_{v}\right), 3 \%$ for the vertical modes $\left(\xi_{w}\right)$ and a $10 \%$ for the torsional modes $\left(\xi_{\varphi}\right)$. Both configurations were analysed for all the angles of attack considered and for the cases of activated and not activated mechanical dampers. The damping constant of the mechanical dampers is $40 \mathrm{kN} \mathrm{s} / \mathrm{m}$.

All cases considered have been calculated with several natural modes, although the critical flutter speed is similar when the analyses were carried out using only the first torsional modes, therefore the flutter instability in this case is dominated by the torsional modes, which are the natural modes with a higher participation in the flutter analysis.

The critical flutter speed obtained is very similar in the two configurations and overall increases with the angle of attack, especially with the positive angles. The inclusion of mechanical dampers significantly affects the critical flutter speed for large angles of attack although it has a lesser impact in the critical flutter speed for the small angles of attack.

\section{ACKNOWLEDGEMENTS}

This work has been funded by the Spanish Minister of Science and Innovation with reference PID2019-110786GB-I00 and the Galician regional government with reference ED431C2017/72. The collaboration in the wind tunnel test of the Research Assistant Miguel Roca is recognized.

\section{REFERENCES}

[1] Jurado, J.Á. \& Hernández, S., Sensitivity analysis of bridge flutter with respect to mechanical parameters of the deck. Structural and Multidisciplinary Optimization, 27(4), pp. 272-283, 2004.

[2] Jurado, J.Á., Hernández, S., Nieto, F. \& Mosquera, A., Bridge Aeroelasticity: Sensitivity Analysis and Optimal Design, WIT Press: Southampton and Boston, 2011.

[3] Kusano, I., Baldomir, A., Jurado, J.A. \& Hernández, S., Reliability based design optimization of long-span bridges considering flutter. Journal of Wind Engineering and Industrial Aerodynamics, 135, pp. 149-162, 2014. 
[4] Jurado, J.Á., Kusano, I., Hernández, S. \& Nieto, F., Improvement of multimodal flutter analysis code, FLAS. EACWE. Proc. European and African Congress in Wind Engineering, Cambridge, UK, 7-11 Jul. 2013.

[5] Chen, A., He, X. \& Xiang, H., Identification of 18 flutter derivatives of bridge decks. Journal of Wind Engineering and Industrial Aerodynamics, 90, pp. 2007-2022, 2002.

[6] Sarkar, P.P., Jones, N.P. \& Scanlan, R.H., System identification for estimation of flutter derivatives. Journal of Wind Engineering and Industrial Aerodynamics, 41-44, pp. 1243-1254, 1992.

[7] Ibrahim, S.R., Fundamentals of time domain modal identification. Modal Analysis and Testing, eds J.M.M. Silva \& N.M.M. Maia, NATO Science Series (Series E: Applied Sciences), vol 363, Springer: Dordrecht, 1999. DOI: 10.1007/978-94-011-4503-9_11. 